\title{
Curvas de Percentis de Valores Normais de Medidas Ecocardiográficas em Crianças Eutróficas Procedentes da Região Centro-Sul do Estado de São Paulo
}

Percentile Curves of Normal Values of Echocardiographic Measurements in Normal Children from the Central-Southern Region of the State of São Paulo, Brazil

Rossano César Bonatto, José Roberto Fioretto, Katashi Okoshi, Beatriz Bojikian Matsubara, Carlos Roberto Padovani, Thiago Colletti Remond Manfrin, Michelle de Farias Gobbi, Rodolfo Silva De Martino, Edson Antônio Bregagnollo Universidade Estadual de São Paulo - UNESP - Botucatu, SP

Objetivo: Avaliar os valores de medidas ecocardiográficas em crianças eutróficas sem cardiopatia, relacionando-os com a superfície corporal $\left(\mathrm{SC}, \mathrm{m}^{2}\right)$, e construir curvas de percentis que relacionem as variáveis ecocardiográficas estudadas com a SC.

Métodos: Foram analisadas medidas ecocardiográficas unidimensionais de crianças entre 1 e 144 meses de idade. Avaliaramse: diâmetros diastólicos dos ventrículos direito (VDd, mm) e esquerdo (VEd, mm), sistólico do VE (VEs, mm), da via de saída do VD (VSVD, mm), da aorta (DAo, mm) e do átrio esquerdo (DAE, mm); fração de ejeção do VE (FEVE, \%); porcentagem da variação do diâmetro ventricular esquerdo ( $\triangle \mathrm{VE}, \%)$; espessura diastólica do septo interventricular (ESIV, mm) e da parede posterior do VE (EPPVE, mm); massa (MVE, g) e índice de massa muscular do VE (IMVE, g/m²).

Resultados: Ao final do estudo, 595 crianças (326 do sexo masculino) foram avaliadas. Os valores das medidas ecocardiográficas apresentaram boa correlação com a SC e possibilitaram a construção de curvas de percentis (3\%, 25\%, 50\%, 75\% e 97\%). Diferenças estatisticamente significantes, entre os sexos, foram evidenciadas para as variáveis VEs, VEd, VSVD, DAo, MVE e IMVE, sendo os maiores valores observados em crianças do sexo masculino.

Conclusão: As curvas de percentis dos valores obtidas podem ser utilizadas como referência para a avaliação de crianças com suspeita de cardiopatia ou para o acompanhamento daquelas já diagnosticadas como cardiopatas ou em tratamento com agentes potencialmente cardiotóxicos.

Palavras-chave: Ecocardiografia, criança, valores de referência, superfície corporal.

Objective: To asses the values of echocardiographic measurements in normal children without cardiopathy and to build percentile curves, relating them to the body surface ( $\left.B S, m^{2}\right)$.

Methods: We analyzed M-mode echocardiographic measurements for children between one and 144 months of age. We assessed right ventricular diastolic diameter (RVDd, $\mathrm{mm}$ ) and left ventricular diastolic diameter (LVDd, mm), LV systolic diameter (LVSd, mm), right ventricular outflow tract diameter (RVOT, $\mathrm{mm}$ ), aortic diameter ( $\mathrm{OoD}, \mathrm{mm})$ and left atrial diameter (LAD, mm); left ventricular ejection fraction (LVEF, $\%)$; percentage of variation of left ventricular diameter ( $\triangle \mathrm{LV}, \%)$; interventricular septum diastolic thickness (IVSDT, mm) and LV posterior wall diastolic thickness (PWDT, mm); left ventricular mass (LVM, g) and LV mass index (LVMI, g/m²).

Results: At the end of the study, 595 children (326 male) were assessed. The values of echocardiographic measurements showed a good correlation with BS and allowed the constructing of percentile curves (3\%, 25\%, 50\%, 75\% and 97\%). Statistically significant differences between the genders were evidenced for the following variables: LVSd, LVDd, RVOT, AoD, LVM and LVMI, and the highest values were observed in male children.

Conclusion: The percentile curves of the values obtained can be used as a reference to assess children with suspicion of cardiopathy or to follow-up on those with diagnosed cardiopathy or under treatment with potentially cardiotoxic drugs.

Key words: Echocardiography, child, reference values, body surface area.

Correspondência: Rossano César Bonatto •

Depto de Pediatria - Faculdade de Medicina de Botucatu-UNESP - Campus de Rubião Júnior - 18618-000 - Botucatu, SP

E-mail: rbonatto@fmb.unesp.br

Artigo recebido em 22/02/05; revisado recebido em 13/03/06; aceito em 11/04/06. 
A ecocardiografia permite avaliação não-invasiva de dimensões e da anatomia das estruturas cardíacas, assim como de características funcionais do coração $^{1,2}$. Tais aspectos tornaram esse exame fundamental para diagnóstico, avaliação das repercussões e acompanhamento de crianças e adolescentes com suspeita ou portadores de cardiopatia ${ }^{2-6}$. Vários estudos utilizaram a ecocardiografia para estabelecer padrões de normalidade das medidas cardíacas na população ${ }^{7-}$ 13.

Atualmente, os valores utilizados como referência de normalidade das dimensões cardíacas em crianças são derivados de estudos das décadas de 1970 e 1980, que incluíram pequena amostra de lactentes, pré-escolares e adolescentes ${ }^{1,5,14-18}$. Sabe-se que a principal limitação de estudos que utilizam amostras populacionais de crianças para obter valores de normalidade é que os resultados podem ser influenciados pelo número de indivíduos e pelas características da população estudada ${ }^{19}$. Assim sendo, é necessário estabelecer valores de referência a partir de um maior número de crianças, incluindo todas as faixas etárias, e levar em conta que fatores raciais podem influenciar os valores das medidas cardíacas.

Em nosso país, a maioria dos serviços de ecocardiografia ${ }^{20,21}$ utiliza padrões norte-americanos como referência para os valores de medidas ecocardiográficas em crianças, o que pode induzir a erros de interpretação, visto que fatores ambientais, socioeconômicos e raciais podem influenciar os padrões antropométricos de uma população ${ }^{22}$.

Os objetivos deste estudo foram estabelecer os valores de medidas ecocardiográficas em uma amostra de crianças eutróficas e sadias, correlacionando-os com a superfície corporal (SC), e construir curvas de percentis que relacionem as variáveis ecocardiográficas estudadas com a SC.

\section{Métodos}

O estudo foi aprovado pelo Comitê de Ética em Pesquisa da instituição.

Parte deste estudo teve financiamento de bolsa de iniciação científica do CNPq.

Foram estudadas crianças eutróficas entre 1 e 144 meses de idade, atendidas em serviço de Cardiologia Pediátrica, no período de janeiro de 1991 a dezembro de 2001, procedentes da região Centro-Sul do Estado de São Paulo. Os pacientes não tinham doença cardíaca ou história de envolvimento do coração por quadros infecciosos, doença neuromuscular ou distúrbios metabólicos, além de não serem portadores de nenhuma característica fenotípica de síndrome genética. Eram crianças atendidas em consulta por sopro cardíaco e que apresentaram exames eletrocardiográficos e radiológicos normais. O exame ecocardiográfico foi realizado para afastar definitivamente o diagnóstico de cardiopatia.

Dados referentes a sexo, idade, peso, estatura e valores das medidas ecocardiográficas foram coletados no dia da realização do exame ecocardiográfico. O peso e a estatura foram utilizados para o cálculo da SC em metro quadrado $\left(\mathrm{m}^{2}\right)$, obtida pela fórmula de DuBois \& DuBois ${ }^{23}$. O índice de massa corporal (IMC), em quilograma por metro quadrado $\left(\mathrm{kg} / \mathrm{m}^{2}\right)$, foi calculado pelo índice de Quetelet ${ }^{24}$.

Crianças com desnutrição energético-protéica (DEP) ${ }^{25,26} \mathrm{e}$ obesas $^{25,26}$ foram excluídas, visto que essas condições podem influenciar as medidas cardíacas ${ }^{27-34}$.

Os exames ecocardiográficos foram realizados por dois ecocardiografistas, sendo $85 \%$ realizados por um deles. A variação interobservador verificada no Serviço de Ecocardiografia do hospital é menor de 10\%, e a variação intra-observador, menor de $5 \%$ para os valores das medidas ecocardiográficas utilizadas.

As imagens ecocardiográficas foram obtidas nas posições precordiais padronizadas ${ }^{1,35}$, seguindo-se as recomendações da American Society of Echocardiography ${ }^{36}$. Foram utilizados dois equipamentos ecocardiográficos: um fabricado pela Advanced Technology Laboratories, modelo Ultramark $8^{\circledR}$, e outro pela Hewlett Packard, modelo Sonos $2000^{\circledR}$, com transdutores de 3,0, 3,5 e/ou 5,0 MHz de acordo com a idade e o peso da criança, com monitorização simultânea de uma derivação eletrocardiográfica. Cerca de $80 \%$ dos exames foram realizados com o segundo equipamento.

Os exames ecocardiográficos foram realizados predominantemente com as crianças despertas e tranqüilas. Quando necessário, procedia-se a sedação com hidrato de cloral na dose de 20 a $40 \mathrm{mg} / \mathrm{kg}$, administrado por via oral, dose que não exerce ação cardiovascular importante ${ }^{37}$.

Após exame ecocardiográfico padrão completo descartando doença cardíaca, obteve-se o corte paraesternal transversal para a realização das medidas a serem utilizadas na construção das curvas de percentis. Os valores das medidas ecocardiográficas e suas derivadas foram analisados no modo unidimensional, após as imagens terem sido direcionadas pelo modo bidimensional (figs. 1 e 2). Considerou-se a média de três medidas de cada variável ecocardiográfica avaliadas em tempo real (on-line). As medidas utilizadas foram: diâmetro diastólico do ventrículo direito (VDd, mm), diâmetros sistólico (VEs, $\mathrm{mm}$ ) e diastólico (VEd, mm) do ventrículo esquerdo, fração de ejeção do ventrículo esquerdo (FEVE, \%), porcentagem da variação do diâmetro ventricular esquerdo ( $\triangle \mathrm{VE}, \%)$, espessura diastólica do septo interventricular (ESIV, mm) e da parede posterior do ventrículo esquerdo (EPPVE, mm), diâmetro da via de saída do ventrículo direito (VSVD, mm), diâmetro da aorta (DAo, mm), diâmetro do átrio esquerdo (DAE, mm), massa (MVE, g) e índice de massa muscular do VE (IMVE, $\mathrm{g} / \mathrm{m}^{2}$ ). A massa muscular do VE (MVE) foi estimada pela fórmula da Penn Convention ${ }^{38}$ corrigida para crianças $^{6}$ e o índice de massa do VE (IMVE) foi obtido dividindo-se a MVE pela SC. Para o cálculo da $\triangle V E$ e da FEVE foram utilizadas as seguintes fórmulas: $\triangle \mathrm{VE}(\%)=[(\mathrm{VEd}-\mathrm{VEs}) / \mathrm{VEd}] \times 100 \mathrm{e}$ $\operatorname{FEVE}(\%)=\left[\left(\mathrm{VEd}^{3}-\mathrm{VEs}^{3}\right) / \mathrm{VEd}^{3}\right] \times 100^{39}$.

Os valores das medidas ecocardiográficas foram correlacionados com a SC e possibilitaram construir curvas de percentis (3\%, 25\%, 50\%, 75\% e 97\%). Foram utilizadas, no mínimo, as medidas ecocardiográficas de 20 crianças em cada faixa de SC para a construção das curvas de percentis.

Análise estatística - Para comparação entre os sexos utilizouse o teste $t$ de Student ${ }^{40}$.

As associações das medidas ecocardiográficas em relação a SC, peso, estatura e idade foram realizadas utilizando-se 
Bonatto e cols.

CURVAS DE PERCENTIS DE VALORES NORMAIS DE MEDIDAS ECOCARDIOGRÁFICAS EM

CRIANÇAS EUTRÓFICAS PROCEDENTES DA REGIÃO CENTRO-SUL DO ESTADO DE SÃO PAULO

\section{Artigo Original}

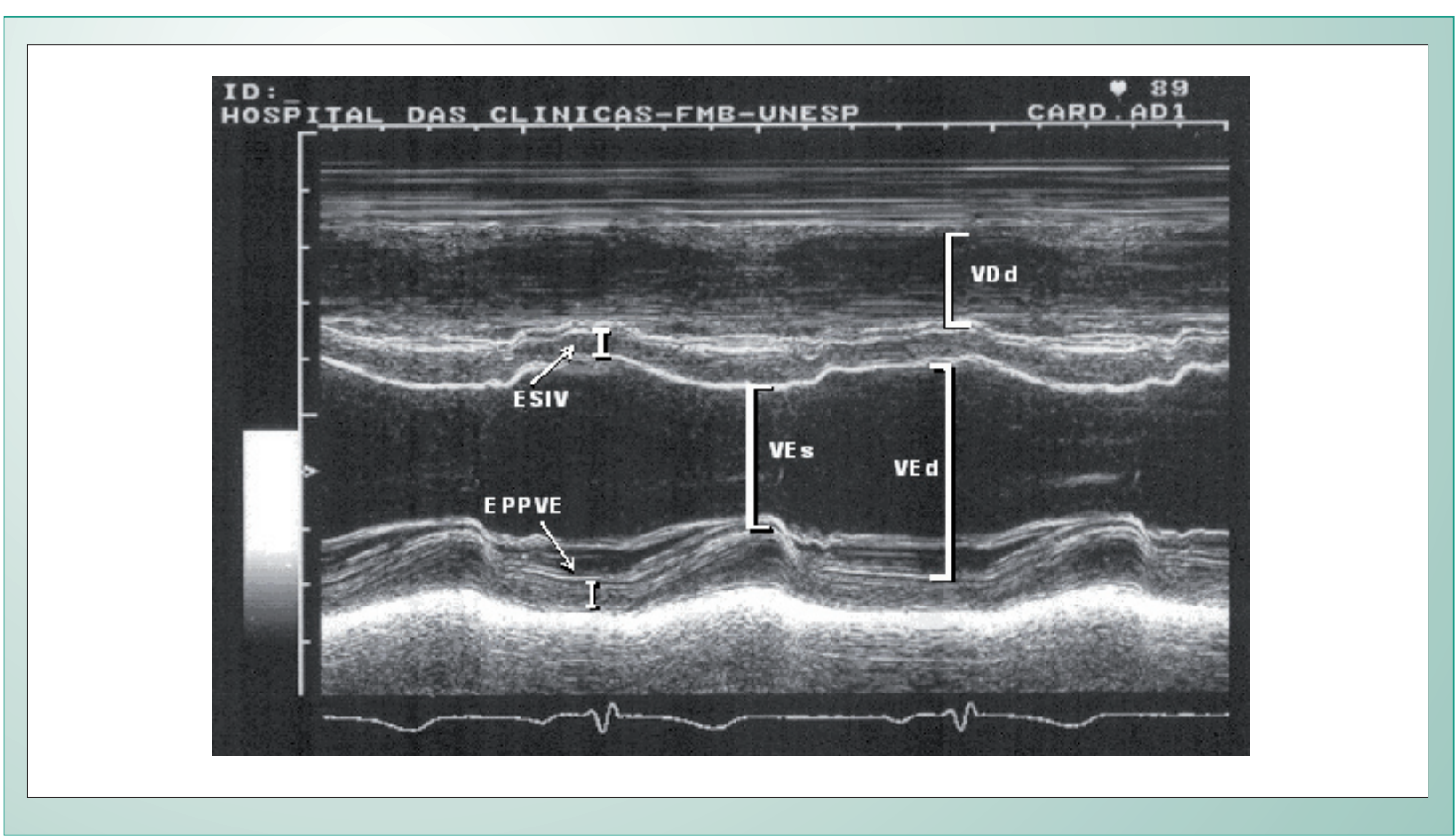

Fig. 1 - Imagem ilustrativa mostrando os locais de medida dos diâmetros diastólicos dos ventrículos direito (VDd) e esquerdo (VEd), diâmetro sistólico do VE (VEs), e espessuras diastólicas do septo interventricular (ESIV) e da parede posterior do VE (EPPVE).

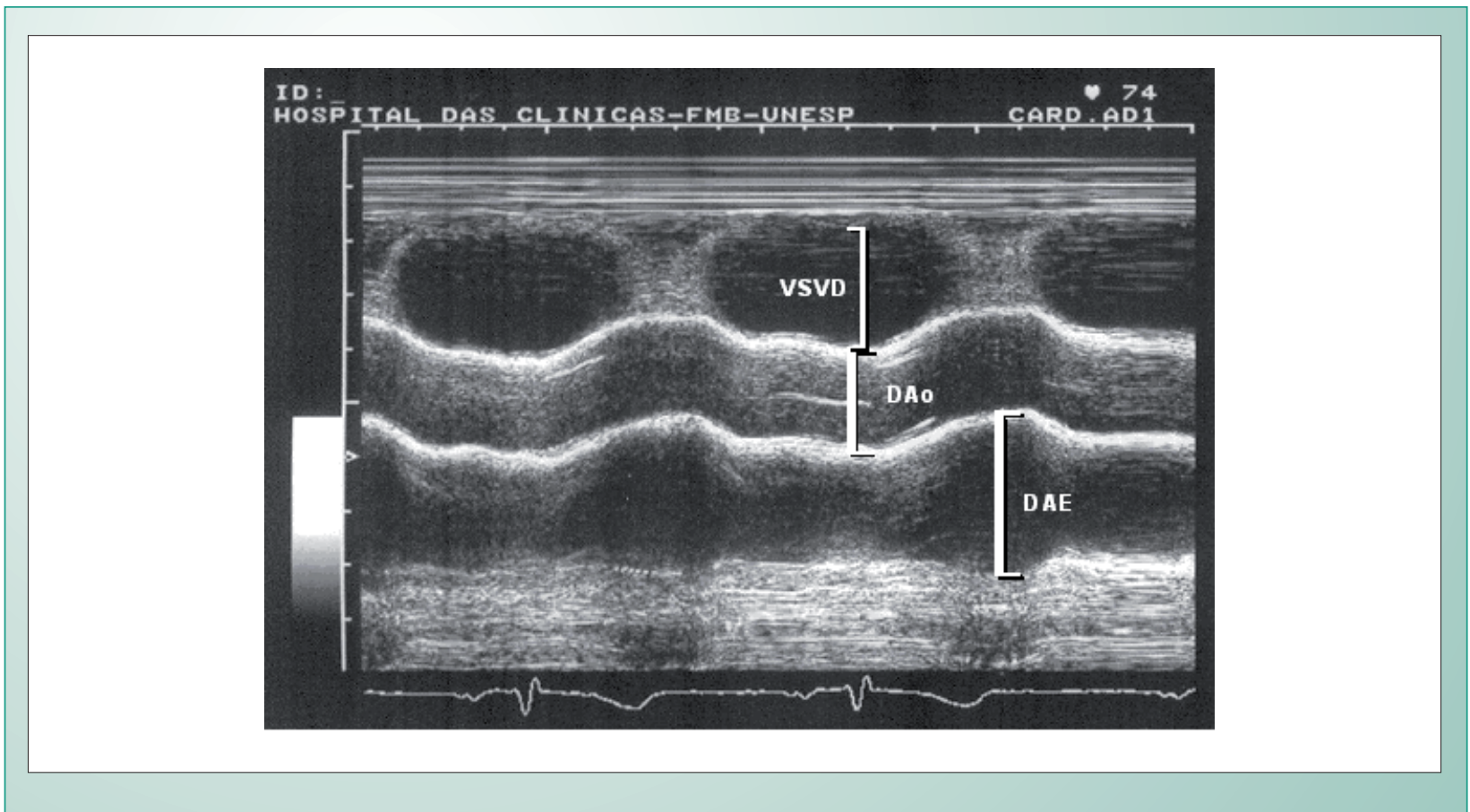

Fig. 2 - Imagem ilustrativa mostrando os locais de medida dos diâmetros da via de saída do ventrículo direito (VSVD), da aorta (DAo) e do átrio esquerdo (DAE).

o Coeficiente de Correlação de Pearson e o respectivo teste estatístico para igualdade dos coeficientes obtidos no dois $\operatorname{sexos}^{40}$. Complementou-se o estudo do perfil das variáveis ecocardiográficas com a construção de curvas de percentis, destacando-se os percentis 3\%, 25\%, 50\%, 75\% e 97\%.

Considerando tratar-se de estudo transversal, foi estipulado número mínimo de 500 crianças para um erro amostral de $2 \%$.
Todas as análises estatísticas foram efetuadas considerando-se o nível de significância de 5\% ( $<<0,05)$.

\section{Resultados}

Ao final do estudo, 595 crianças preencheram os critérios de inclusão, sendo 326 (54,8 \%) do sexo masculino e 269 
$(45,2 \%)$ do feminino. A sedação foi necessária em cerca de $10 \%$ das crianças, predominantemente na faixa etária de seis meses a dois anos. A variação interobservador foi de $8 \%$, e intra-observador, de $5 \%$ para os valores das medidas ecocardiográficas utilizadas.

O peso variou de 3,4 a $53,0 \mathrm{~kg}$; a altura, de 0,51 a 1,60 metros; e a SC, de 0,20 a 1,53 $\mathrm{m}^{2}$. Na tabela 1 são apresentados os valores médios e seus respectivos desviospadrão referentes a idade, peso, estatura, SC, IMC e das medidas ecocardiográficas e de suas derivadas. Também são apresentadas a variação e a comparação dessas variáveis em relação ao sexo. Foram observadas diferenças estatisticamente significantes entre os sexos com relação a VEs, VEd, DAo, VSVD, MVE e IMVE.

\begin{tabular}{|c|c|c|c|}
\hline & Geral & Masculino & Feminino \\
\hline & $(n=595)$ & $(n=326)$ & $(\mathrm{n}=269)$ \\
\hline Idade (meses) & $60,9 \pm 40,1$ & $60,8 \pm 39,8$ & $60,9 \pm 40,5$ \\
\hline Peso (kg) & $19,4 \pm 9,3$ & $19,5 \pm 9,3$ & $19,4 \pm 9,4$ \\
\hline Estatura (m) & $1,05 \pm 0,24$ & $1,05 \pm 0,24$ & $1,05 \pm 0,25$ \\
\hline $\mathrm{SC}\left(\mathrm{m}^{2}\right)$ & $0,74 \pm 0,27$ & $0,74 \pm 0,27$ & $0,74 \pm 0,28$ \\
\hline IMC $\left(\mathrm{kg} / \mathrm{m}^{2}\right)$ & $16,6 \pm 1,68$ & $16,6 \pm 1,61$ & $16,6 \pm 1,77$ \\
\hline VDd (mm) & $13,2 \pm 2,9$ & $14,0 \pm 3,8$ & $13,7 \pm 3,5$ \\
\hline VEs $(m m)$ & $20,8 \pm 4,2$ & $21,2 \pm 4,4$ & $20,3 \pm 4,0^{*}$ \\
\hline VEd (mm) & $34,9 \pm 3,5$ & $35,5 \pm 6,2$ & $34,1 \pm 5,8^{*}$ \\
\hline ESIV (mm) & $5,3 \pm 0,9$ & $5,4 \pm 0,9$ & $5,3 \pm 0,8$ \\
\hline EPPVE (mm) & $5,3 \pm 0,9$ & $5,4 \pm 0,9$ & $5,3 \pm 0,9$ \\
\hline VSVD (mm) & $19,5 \pm 3,9$ & $19,9 \pm 4,0$ & $18,9 \pm 3,6^{*}$ \\
\hline DAo (mm) & $18,5 \pm 3,6$ & $18,9 \pm 3,7$ & $17,9 \pm 3,3^{*}$ \\
\hline $\mathrm{DAE}(\mathrm{mm})$ & $25,7 \pm 4,7$ & $26,0 \pm 4,8$ & $25,3 \pm 4,7$ \\
\hline MVE (g) & $58,0 \pm 26,8$ & $60,6 \pm 28,3$ & $54,8 \pm 24,6^{*}$ \\
\hline IMVE $\left(\mathrm{g} / \mathrm{m}^{2}\right)$ & $76,6 \pm 15,1$ & $79,2 \pm 15,0$ & $73,5 \pm 14,6^{*}$ \\
\hline FEVE (\%) & $78,5 \pm 5,4$ & $78,5 \pm 5,6$ & $78,5 \pm 5,1$ \\
\hline$\triangle \mathrm{VE}(\%)$ & $40,5 \pm 5,0$ & $41,0 \pm 5,0$ & $40,0 \pm 5,0$ \\
\hline \multicolumn{4}{|c|}{$\begin{array}{l}\text { * p < 0,05 (sexo masculino vs sexo feminino); SC- superfície corporal; } \\
\text { IMC- índice de massa corporal; VDd- diâmetro do ventrículo direito; } \\
\text { VES: diâmetro sistólico do ventrículo esquerdo; VEd- diâmetro } \\
\text { diastólico do ventrículo esquerdo; ESIV- espessura diastólica do septo } \\
\text { interventricular; EPPVE- espessura diastólica da parede posterior do } \\
\text { ventrículo esquerdo; VSVD- diâmetro da via de saída do ventrículo } \\
\text { direito; DAo: diâmetro da aorta; DAE- diâmetro do átrio esquerdo; } \\
\text { MVE- massa do ventrículo esquerdo; IMVE- índice de massa do } \\
\text { ventrículo esquerdo; FEVE- fração de ejeção do ventrículo esquerdo; } \\
\Delta V E \text { - porcentagem de variação do diâmetro ventricular esquerdo; } \\
\text { n- número de crianças; kg- kilograma; g- grama; m- metro; mm- } \\
\text { milímetros; \% = porcentagem. }\end{array}$} \\
\hline \multicolumn{4}{|c|}{$\begin{array}{l}\text { Tabela } 1 \text { - Valores das médias e desvios-padrão da idade, medidas } \\
\text { antropométricas e ecocardiográficas de todas as crianças avaliadas } \\
\text { e separadas de acordo com o sexo }\end{array}$} \\
\hline
\end{tabular}

Na tabela 2 estão relacionados os valores dos coeficientes de correlação de Pearson das medidas ecocardiográficas em relação a peso, estatura, idade e SC de todas as crianças estudadas e de acordo com o sexo. Com exceção do IMVE, da
FEVE e da $\triangle V E$, todas as medidas ecocardiográficas analisadas apresentaram boa correlação com peso, altura, SC e idade. Nenhuma das medidas ecocardiográficas avaliadas neste estudo apresentou boa correlação com o IMC. Os coeficientes de correlação de Pearson entre os valores das medidas ecocardiográficas e idade, peso, estatura, SC e sexo não apresentaram diferenças estatísticas significantes entre si.

Na tabela 3 estão expressos os valores dos percentis 3\%, $50 \%$ e $97 \%$ de cada variável ecocardiográfica analisada para a SC ajustada a cada $0,1 \mathrm{~m}^{2}$ a partir de $0,2 \mathrm{~m}^{2}$, considerando-se todas as crianças estudadas.

Nas tabelas 4 e 5 estão relacionados os valores dos percentis 3\%, 50\% e 97\% das variáveis ecocardiográficas que apresentaram diferenças estatísticas significantes entre os sexos, considerando-se isoladamente as crianças do sexo masculino (tab. 4) e do sexo feminino (tab. 5).

As figuras 3 a 11 mostram as curvas de percentis (3\%, 25\%, $50 \%, 75 \%$ e $97 \%$ ) das variáveis ecocardiográficas analisadas em relação à SC, considerando-se todas as crianças do estudo.

\section{Discussão}

Atualmente, os valores de referência de medidas ecocardiográficas de crianças que são utilizados em nosso meio são baseados em padrões da normalidade de populações de outros países ${ }^{1,16}$, sendo importante estabelecer padrões nacionais.

A análise dos valores das medidas ecocardiográficas de acordo com o sexo mostrou diferenças significativas quanto a VEs, VEd, VSVD, DAo, MVE e IMVE, com valores maiores no sexo masculino. Considerando que não houve diferenças em relação a faixa etária, antropometria e suas derivadas estudadas, é possível concluir que essas diferenças estejam relacionadas com o sexo, à semelhança de outros estudos ${ }^{41-}$ ${ }^{43}$. Lester e cols. ${ }^{41}$ estudaram 202 pacientes de 25 dias a 23 anos de idade, norte-americanos, sendo 125 do sexo masculino (69 brancos e 56 negros) e 77 do sexo feminino (34 brancas e 43 negras). Os autores observaram valores das medidas ecocardiográficas significantemente maiores no sexo masculino, quando a SC era maior que $1 \mathrm{~m}^{2}$. Também foram observadas diferenças significativas entre brancos e negros, com dimensões cardíacas maiores entre os negros. Nagasawa e cols. ${ }^{42}$ estudaram 437 crianças japonesas, 264 do sexo masculino e 173 do feminino, na faixa etária entre um mês e 17 anos, com histórico de doença de Kawasaki sem lesões coronárias.

Durante 6,7 anos de acompanhamento clínico, foram realizados 1.595 exames ecocardiográficos nesse grupo de pacientes. Analisando a velocidade do aumento do VEd, os autores observaram valores semelhantes na velocidade de crescimento e de aumento na dimensão do VEd em ambos os sexos, até os 10 anos de idade. Após essa idade, os valores do VEd foram significantemente maiores no sexo masculino. Em 2002, Nagasawa e Arakaki ${ }^{43}$ avaliaram as espessuras diastólica e sistólica do septo interventricular e da parede posterior do VE de 251 crianças japonesas, sendo 128 do sexo masculino e 123 do feminino, na faixa etária entre um mês e 18 anos. 


\begin{tabular}{|c|c|c|c|c|c|c|c|c|c|c|c|c|}
\hline \multirow{2}{*}{$\begin{array}{c}\text { Medida ecocardio- } \\
\text { gráfica }\end{array}$} & \multicolumn{3}{|c|}{ Idade } & \multicolumn{3}{|c|}{ Peso } & \multicolumn{3}{|c|}{ Estatura } & \multicolumn{3}{|c|}{ SC } \\
\hline & G & $\mathbf{M}$ & $\mathbf{F}$ & G & $\mathbf{M}$ & $\mathbf{F}$ & G & $\mathbf{M}$ & $F$ & G & $\mathbf{M}$ & $F$ \\
\hline VDd & 0,63 & 0,63 & 0,64 & 0,64 & 0,64 & 0,65 & 0,63 & 0,64 & 0,64 & 0,64 & 0,64 & 0,65 \\
\hline VEs & 0,82 & 0,84 & 0,85 & 0,81 & 0,83 & 0,83 & 0,82 & 0,84 & 0,85 & 0,82 & 0,84 & 0,84 \\
\hline VEd & 0,88 & 0,91 & 0,91 & 0,89 & 0,91 & 0,91 & 0,90 & 0,92 & 0,92 & 0,90 & 0,92 & 0,92 \\
\hline ESIV & 0,80 & 0,83 & 0,82 & 0,81 & 0,84 & 0,83 & 0,81 & 0,84 & 0,83 & 0,81 & 0,84 & 0,83 \\
\hline EPPVE & 0,83 & 0,87 & 0,87 & 0,84 & 0,87 & 0,87 & 0,84 & 0,87 & 0,87 & 0,84 & 0,87 & 0,88 \\
\hline VSVD & 0,71 & 0,75 & 0,74 & 0,72 & 0,76 & 0,76 & 0,71 & 0,76 & 0,76 & 0,72 & 0,76 & 0,76 \\
\hline DAo & 0,86 & 0,86 & 0,85 & 0,86 & 0,85 & 0,84 & 0,87 & 0,86 & 0,85 & 0,87 & 0,86 & 0,85 \\
\hline DAE & 0,79 & 0,81 & 0,79 & 0,81 & 0,82 & 0,81 & 0,80 & 0,82 & 0,80 & 0,81 & 0,82 & 0,81 \\
\hline MVE & 0,91 & 0,93 & 0,93 & 0,92 & 0,93 & 0,93 & 0,92 & 0,94 & 0,94 & 0,92 & 0,94 & 0,94 \\
\hline IMVE & 0,33 & 0,43 & 0,44 & 0,32 & 0,41 & 0,42 & 0,32 & 0,42 & 0,43 & 0,32 & 0,42 & 0,42 \\
\hline \multicolumn{13}{|c|}{$\begin{array}{l}\text { SC- superfície corporal; VDd- diâmetro do ventrículo direito; VES: diâmetro sistólico do ventrículo esquerdo; VEd- diâmetro diastólico do ventrículo } \\
\text { esquerdo; ESIV- espessura diastólica do septo interventricular; EPPVE- espessura diastólica da parede posterior do ventrículo esquerdo; VSVD- diâmetro da } \\
\text { via de saída do ventrículo direito; DAo: diâmetro da aorta; DAE- diâmetro do átrio esquerdo; MVE- massa do ventrículo esquerdo; IMVE- índice de massa } \\
\text { do ventrículo esquerdo. }\end{array}$} \\
\hline
\end{tabular}

Os pacientes eram eutróficos e sem cardiopatia, e foram submetidos a exame ecocardiográfico por sopro cardíaco inocente. Os autores relataram que os valores de algumas medidas ecocardiográficas foram significantemente maiores no sexo masculino, principalmente a partir de $1 \mathrm{~m}^{2}$ de SC.

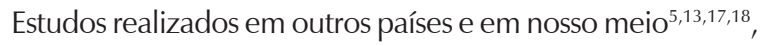
contudo, não encontraram diferenças significantes entre os valores das medidas cardíacas quando se compararam os sexos masculino e feminino. Os estudos nacionais ${ }^{5,17,18}$ avaliaram crianças em mesma faixa etária de nosso estudo, porém incluíram amostras com pequeno número de crianças. Assim, ainda é controverso se existe diferença entre os valores das medidas cardíacas obtidas por ecocardiografia quando se comparam os sexos masculino e feminino. Nosso estudo incluiu amostra populacional representativa com maior número de crianças em cada faixa etária analisada em relação aos estudos anteriormente $\operatorname{citados}^{5,17,18}$, o que provavelmente possibilitou identificar diferenças significantes entre os sexos.

A diferença dos valores das médias do VEs, VEd, DAo e VSVD entre os sexos, no entanto, é pequena (tab. 2), com valores maiores no sexo masculino. Apesar de as diferenças entre os valores das medidas ecocardiográficas serem estatisticamente significantes, do ponto de vista biológico pode-se admitir que essa diferença tem pouca relevância, sendo difícil considerá-la no dia-a-dia.

Não foram observadas diferenças significativas entre os valores dos coeficientes de correlação de Pearson quando se correlacionaram os valores das medidas ecocardiográficas estudadas com idade, peso, estatura e SC. O mesmo foi observado quando os valores dos coeficientes de Pearson foram analisados de acordo com o sexo, o que está em conformidade com os estudos de Rogé e cols. ${ }^{1}$ e Kampmann e cols. ${ }^{13}$.
Não há consenso na literatura sobre qual parâmetro antropométrico apresenta melhor correlação com as medidas cardíacas avaliadas por meio da ecocardiografia. Alguns estudos mostraram melhor correlação com a SC ${ }^{6,34}$ e outros com a estatura ${ }^{5,17,18}$. Em nosso estudo, evidenciamos que, para crianças eutróficas e sadias, é indiferente correlacionar os valores das medidas ecocardiográficas com idade, peso, estatura ou SC.

Utilizamos a SC como variável independente, visto que: não houve diferenças significantes dos valores dos coeficientes de correlação entre os valores das medidas ecocardiográficas e idade, peso, estatura e SC; as medidas hemodinâmicas habitualmente são expressas em relação à SC; para determinação do estado nutricional há necessidade do conhecimento do peso e estatura que entram no cálculo da SC; os equipamentos atuais calculam a SC e vários estudos mostram boa correlação entre dimensões cardíacas e $\mathrm{SC}^{13}$.

A comparação das médias dos percentis 3\% e 97\% dos valores das medidas ecocardiográficas deste estudo com as referências mais utilizadas ${ }^{1,16}$ mostra que os valores das medidas ecocardiográficas são próximos. No entanto, o número de indivíduos estudados por esses autores ${ }^{1,16}$ foi bem menor que o número de crianças que nós incluímos. Além disso, o estudo de Rogé e cols. ${ }^{1}$ foi realizado antes da publicação das recomendações da American Society of Echocardiography ${ }^{36}$.

Os valores das medidas ecocardiográficas encontrados no presente estudo são comparáveis aos obtidos por Kampmann e cols. ${ }^{13}$. Tal semelhança, possivelmente, se deve à inclusão de pacientes eutróficos nos dois estudos, em que pesem as prováveis diferenças raciais entre as duas populações. Também, é provável que, independentemente das diferenças raciais, as dimensões cardíacas sejam proporcionais à SC. Os limites das curvas de percentis e os valores médios das medidas 


\begin{tabular}{|c|c|c|c|c|c|c|c|c|c|c|c|c|c|}
\hline \multicolumn{2}{|c|}{$\mathrm{SC}(\mathrm{m} 2)$} & \multirow{2}{*}{$\begin{array}{c}\begin{array}{c}\text { VDd } \\
(\mathrm{mm})\end{array} \\
9,0\end{array}$} & \multirow{2}{*}{$\begin{array}{c}\begin{array}{c}\text { VEs } \\
(\mathrm{mm})\end{array} \\
19,5\end{array}$} & \multirow{2}{*}{$\begin{array}{c}\begin{array}{c}\text { VEd } \\
(\mathrm{mm})\end{array} \\
32,0\end{array}$} & \multirow{2}{*}{$\begin{array}{c}\text { FEVE } \\
(\%) \\
81,9\end{array}$} & \multirow{2}{*}{$\begin{array}{c}\Delta \text { VE } \\
(\%) \\
43,0\end{array}$} & \multirow{2}{*}{$\begin{array}{r}\begin{array}{r}\text { ESIV } \\
(\mathrm{mm})\end{array} \\
3,7\end{array}$} & \multirow{2}{*}{$\begin{array}{r}\begin{array}{r}\text { EPPVE } \\
(\mathrm{mm})\end{array} \\
3,7\end{array}$} & \multirow{2}{*}{$\begin{array}{r}\text { MVE (g) } \\
12,0\end{array}$} & \multirow{2}{*}{$\begin{array}{c}\begin{array}{c}\text { IMVE } \\
(\mathrm{g} / \mathrm{m} 2)\end{array} \\
53,5\end{array}$} & \multirow{2}{*}{$\begin{array}{r}\begin{array}{r}\text { VSVD } \\
(\mathrm{mm})\end{array} \\
13,0\end{array}$} & \multirow{2}{*}{$\begin{array}{c}\begin{array}{c}\text { DAo } \\
(\mathrm{mm})\end{array} \\
11,5\end{array}$} & \multirow{2}{*}{$\begin{array}{r}\begin{array}{r}\text { DAE } \\
(\mathrm{mm})\end{array} \\
16,0\end{array}$} \\
\hline 0,2 & P50 & & & & & & & & & & & & \\
\hline \multirow{3}{*}{0,3} & P3 & 9,3 & 17,3 & 29,0 & 66,8 & 31,0 & 3,4 & 3,5 & 12,7 & 44,3 & 11,0 & 11,0 & 14,0 \\
\hline & P50 & 11,9 & 19,8 & 32,3 & 80,8 & 42,0 & 4,0 & 4,0 & 19,7 & 65,2 & 13,5 & 13,0 & 18,0 \\
\hline & P97 & 14,5 & 21,6 & 35,5 & 88,7 & 52,0 & 4,9 & 4,5 & 32,0 & 101 & 16,9 & 15,0 & 22,0 \\
\hline \multirow{3}{*}{0,4} & P3 & 9,5 & 17,5 & 29,3 & 74,6 & 37,0 & 3,6 & 3,7 & 20,6 & 50,7 & 12,5 & 12,0 & 16,0 \\
\hline & P50 & 12,3 & 19,9 & 32,9 & 79,9 & 41,0 & 4,3 & 4,3 & 28,3 & 70,4 & 15,0 & 15,0 & 21,0 \\
\hline & P97 & 14,9 & 21,8 & 36,2 & 86,2 & 48,0 & 5,3 & 5,0 & 43,4 & 104 & 20,0 & 17,0 & 25,4 \\
\hline \multirow{3}{*}{0,5} & P3 & 9,6 & 17,5 & 29,6 & 71,7 & 34,0 & 3,7 & 3,9 & 25,7 & 52,1 & 13,4 & 13,0 & 18,0 \\
\hline & P50 & 12,6 & 20,0 & 32,9 & 80,4 & 42,0 & 4,6 & 4,7 & 35,6 & 69,8 & 17,0 & 16,0 & 23,0 \\
\hline & P97 & 15,8 & 21,9 & 36,4 & 87,5 & 50,0 & 5,7 & 5,5 & 51,0 & 98,6 & 22,0 & 18,7 & 27,0 \\
\hline \multirow{3}{*}{0,6} & P3 & 9,7 & 17,9 & 29,8 & 69,3 & 33,0 & 4,0 & 4,2 & 29,2 & 50,7 & 14,5 & 14,0 & 20,0 \\
\hline & P50 & 12,7 & 20,1 & 33,1 & 79,1 & 41,0 & 5,0 & 5,0 & 45,3 & 75,8 & 18,0 & 17,0 & 24,5 \\
\hline & P97 & 17,0 & 22,7 & 36,8 & 85,2 & 47,0 & 6,0 & 5,9 & 63,8 & 106,4 & 23,0 & 20,6 & 29,0 \\
\hline \multirow{3}{*}{0,7} & P3 & 9,8 & 18,0 & 30,0 & 67,5 & 31,0 & 4,2 & 4,4 & 35,8 & 46,8 & 15,5 & 15,0 & 21,5 \\
\hline & P50 & 13,0 & 20,7 & 33,4 & 77,8 & 39,0 & 5,2 & 5,4 & 52,7 & 74,0 & 19,0 & 18,0 & 26,0 \\
\hline & P97 & 18,1 & 23,8 & 37,5 & 86,3 & 48,0 & 6,2 & 6,2 & 73,0 & 99,0 & 25,0 & 22,0 & 31,0 \\
\hline \multirow{3}{*}{0,8} & P3 & 9,8 & 18,0 & 31,1 & 68,8 & 32,0 & 4,3 & 4,7 & 41,9 & 49,8 & 16,5 & 16,0 & 22,5 \\
\hline & P50 & 13,4 & 21,3 & 34,6 & 79,0 & 41,0 & 5,5 & 5,7 & 64,5 & 79,1 & 20,0 & 19,0 & 27,5 \\
\hline & P97 & 19,2 & 24,7 & 39,0 & 86,5 & 49,0 & 6,5 & 6,6 & 83,2 & 105,5 & 26,3 & 23,1 & 32,2 \\
\hline \multirow{3}{*}{0,9} & P3 & 9,9 & 18,0 & 31,2 & 68,8 & 32,0 & 4,8 & 4,9 & 52,6 & 57,9 & 17,0 & 17,0 & 24,0 \\
\hline & P50 & 13,8 & 21,8 & 35,7 & 79,0 & 41,0 & 5,9 & 5,9 & 71,4 & 79,0 & 21,0 & 20,0 & 28,5 \\
\hline & P97 & 20,1 & 26,1 & 40,5 & 85,2 & 47,0 & 6,9 & 6,8 & 97,3 & 106,2 & 27,8 & 23,9 & 33,4 \\
\hline \multirow{3}{*}{1,0} & P3 & 9,9 & 18,1 & 31,5 & 68,8 & 32,0 & 5,0 & 5,2 & 58,0 & 55,3 & 17,5 & 18,2 & 25,0 \\
\hline & P50 & 14,2 & 21,9 & 36,1 & 77,3 & 39,0 & 6,2 & 6,1 & 79,7 & 79,7 & 22,0 & 21,0 & 29,5 \\
\hline & P97 & 21,0 & 28,9 & 42,0 & 85,5 & 48,0 & 7,2 & 7,0 & 110,0 & 119,6 & 28,3 & 25,0 & 34,7 \\
\hline \multirow{3}{*}{1,1} & P3 & 9,9 & 18,3 & 31,7 & 62,5 & 28,0 & 5,5 & 5,5 & 69,5 & 62,0 & 18,1 & 19,0 & 26,0 \\
\hline & P50 & 14,8 & 22,6 & 36,4 & 77,3 & 39,0 & 6,3 & 6,4 & 90,3 & 80,2 & 22,5 & 22,0 & 31,0 \\
\hline & P97 & 21,8 & 30,1 & 43,5 & 86,4 & 49,0 & 7,3 & 7,1 & 121,0 & 104,0 & 28,4 & 26,1 & 35,7 \\
\hline & P3 & 10,1 & 18,5 & 31,8 & 68,3 & 32,0 & 5,8 & 5,8 & 78,6 & 62,5 & 18,7 & 20,2 & 27,0 \\
\hline 1,2 & P50 & 15,3 & 23,5 & 37,0 & 78,4 & 40,0 & 6,5 & 6,7 & 101,2 & 82,2 & 23,0 & 23,0 & 32,0 \\
\hline & P97 & 22,3 & 30,4 & 45,5 & 85,9 & 48,0 & 7,5 & 7,2 & 130,1 & 111,2 & 28,7 & 26,8 & 36,5 \\
\hline & P3 & 10,2 & 18,6 & 31,8 & 72,3 & 35,0 & 6,0 & 6,0 & 85,0 & 62,5 & 20,5 & 20,7 & 28,0 \\
\hline 1,3 & P50 & 15,7 & 23,8 & 38,0 & 78,9 & 40,0 & 6,7 & 6,9 & 112,0 & 74,0 & 24,5 & 23,5 & 33,0 \\
\hline & P97 & 22,8 & 30,7 & 47,4 & 88,9 & 52,0 & 7,6 & 7,3 & 138,3 & 113,4 & 29,0 & 27,2 & 37,5 \\
\hline & P3 & 10,5 & 18,7 & 32,5 & 69,3 & 33,0 & 6,1 & 6,5 & 93,7 & 66,5 & 21,0 & 21,0 & 29,5 \\
\hline 1,4 & P50 & 16,4 & 24,2 & 40,0 & 77,9 & 40,0 & 7,0 & 7,0 & 124,0 & 89,9 & 25,0 & 24,2 & 34,0 \\
\hline & P97 & 23,1 & 30,8 & 48,0 & 83,8 & 45,0 & 7,8 & 7,4 & 140,5 & 100,4 & 30,5 & 27,5 & 39,2 \\
\hline 1,5 & P50 & 18,0 & 28,0 & 44,0 & 74,2 & 36,0 & 7,0 & 6,0 & 104,0 & 99,3 & 27,0 & 26,0 & 34,5 \\
\hline $\begin{array}{l}\text { epto ir } \\
\text { lo vent }\end{array}$ & 政 & $\mathrm{ral} ; \mathrm{VD}$ & diâme & ventro & men, & (lî & 年 & tro & orta; $D$ & iâmetrc & trio e & $\begin{array}{l}\text { E- ínc } \\
\text { to. }\end{array}$ & $\begin{array}{l}\text { ca do } \\
\text { massa }\end{array}$ \\
\hline
\end{tabular}




\begin{tabular}{|c|c|c|c|c|c|c|c|}
\hline \multicolumn{2}{|c|}{$\mathrm{SC}(\mathrm{m} 2)$} & \multirow{2}{*}{$\begin{array}{c}\begin{array}{c}\text { VEs } \\
(\mathrm{mm})\end{array} \\
11,0\end{array}$} & \multirow{2}{*}{$\begin{array}{c}\begin{array}{c}\text { Ved } \\
(\mathrm{mm})\end{array} \\
20,0\end{array}$} & \multirow{2}{*}{$\begin{array}{c}\text { MVE } \\
\text { (g) } \\
11,8\end{array}$} & \multirow{2}{*}{$\begin{array}{c}\begin{array}{c}\text { IMVE } \\
(\mathrm{g} / \mathrm{m} 2)\end{array} \\
53,5\end{array}$} & \multirow{2}{*}{$\begin{array}{c}\text { VSVD } \\
(\mathrm{mm})\end{array}$} & \multirow{2}{*}{$\begin{array}{c}\begin{array}{c}\text { DAo } \\
(\mathrm{mm})\end{array} \\
11,0\end{array}$} \\
\hline 0,2 & P50 & & & & & & \\
\hline \multirow{3}{*}{0,3} & P3 & 9,8 & 20,4 & 15,0 & 46,7 & 12,0 & 11,4 \\
\hline & P50 & 13,0 & 24,0 & 19,1 & 65,4 & 14,0 & 13,0 \\
\hline & P97 & 17,0 & 26,6 & 28,6 & 88,2 & 15,0 & 14,0 \\
\hline \multirow{3}{*}{0,4} & P3 & 13,8 & 25,6 & 22,0 & 53,3 & 13,6 & 14,0 \\
\hline & P50 & 16,0 & 28,0 & 27,7 & 71,8 & 15,0 & 15,0 \\
\hline & P97 & 19,4 & 31,6 & 42,6 & 103,0 & 20,0 & 17,0 \\
\hline \multirow{3}{*}{0,5} & P3 & 15,0 & 27,6 & 27,7 & 54,1 & 13,1 & 13,1 \\
\hline & P50 & 17,0 & 30,5 & 35,6 & 71,3 & 18,0 & 16,0 \\
\hline & P97 & 21,0 & 34,0 & 47,7 & 94,2 & 22,0 & 20,9 \\
\hline \multirow{3}{*}{0,6} & P3 & 17,2 & 30,0 & 29,8 & 50,8 & 15,0 & 16,0 \\
\hline & P50 & 20,0 & 34,0 & 47,7 & 77,2 & 18,0 & 17,5 \\
\hline & P97 & 22,0 & 37,0 & 60,6 & 94,1 & 23,0 & 20,0 \\
\hline \multirow{3}{*}{0,7} & P3 & 17,0 & 31,0 & 39,5 & 57,7 & 15,7 & 14,1 \\
\hline & P50 & 22,0 & 36,0 & 55,3 & 78,3 & 19,0 & 18,0 \\
\hline & P97 & 26,0 & 40,0 & 75,1 & 105,3 & 23,0 & 22,0 \\
\hline \multirow{3}{*}{0,8} & P3 & 19,0 & 34,0 & 50,1 & 65,0 & 18,0 & 17,0 \\
\hline & P50 & 22,5 & 37,3 & 67,4 & 85,6 & 21,0 & 20,0 \\
\hline & P97 & 27,0 & 41,0 & 83,5 & 105,9 & 27,0 & 22,0 \\
\hline \multirow{3}{*}{0,9} & P3 & 18,5 & 33,0 & 53,9 & 59,0 & 18,0 & 18,0 \\
\hline & P50 & 23,0 & 39,0 & 72,9 & 82,6 & 21,0 & 21,0 \\
\hline & P97 & 26,0 & 43,0 & 101,3 & 107,9 & 29,0 & 24,0 \\
\hline \multirow{3}{*}{1,0} & P3 & 20,0 & 36,7 & 68,1 & 67,2 & 18,4 & 17,2 \\
\hline & P50 & 25,0 & 41,0 & 84,9 & 85,3 & 23,0 & 22,0 \\
\hline & P97 & 29,3 & 46,3 & 117,6 & 114,2 & 28,0 & 25,0 \\
\hline \multirow{3}{*}{1,1} & P3 & 22,0 & 37,8 & 76,1 & 67,6 & 18,0 & 20,0 \\
\hline & P50 & 26,5 & 43,0 & 93,6 & 85,7 & 22,0 & 23,5 \\
\hline & P97 & 30,0 & 46,3 & 114,9 & 104,3 & 27,0 & 28,3 \\
\hline \multirow{3}{*}{1,2} & P3 & 22,5 & 39,3 & 79,5 & 65,2 & 19,1 & 21,5 \\
\hline & P50 & 27,5 & 44,5 & 102,8 & 85,3 & 23,5 & 24,0 \\
\hline & P97 & 30,0 & 47,5 & 129,1 & 108,0 & 27,0 & 27,5 \\
\hline \multirow{3}{*}{1,3} & P3 & 23,2 & 42,4 & 92,3 & 69,5 & 23,4 & 22,0 \\
\hline & P50 & 27,5 & 46,0 & 122,2 & 93,6 & 27,0 & 24,5 \\
\hline & P97 & 30,0 & 48,8 & 141,6 & 110,6 & 30,6 & 28,2 \\
\hline \multirow{3}{*}{1,4} & P3 & 23,5 & 41,5 & 95,2 & 68,9 & 23,5 & 24,1 \\
\hline & P50 & 29,5 & 47,5 & 128,3 & 94,7 & 27,5 & 26,0 \\
\hline & P97 & 30,9 & 48,9 & 139,7 & 100,1 & 30,9 & 27,0 \\
\hline 1,5 & P50 & 28,5 & 47,5 & 130,0 & 84,6 & 31,5 & 27,0 \\
\hline \multicolumn{8}{|c|}{$\begin{array}{l}\text { SC- superfície corporal; VES- diâmetro sistólico do ventrículo esquerdo; } \\
\text { VEd- diâmetro diastólico do ventrículo esquerdo; MVE- massa do } \\
\text { ventrículo esquerdo; IMVE- índice de massa do ventrículo esquerdo; } \\
\text { VSVD- diâmetro da via de saída do ventrículo direito; DAo- diâmetro } \\
\text { da aorta. } \\
\text { Tabela } 4 \text { - Percentis } \mathbf{3 \%}, \mathbf{5 0 \%} \text { e } \mathbf{9 7 \%} \text { dos valores das medidas } \\
\text { ecocardiográficas que apresentaram diferenças estatisticamente } \\
\text { significativas entre os sexos, em relação à superfície corporal das } \\
\text { crianças do sexo masculino }\end{array}$} \\
\hline
\end{tabular}

\begin{tabular}{|c|c|c|c|c|c|c|c|}
\hline \multicolumn{2}{|c|}{$\begin{array}{c}\text { SC } \\
(\mathrm{m} 2)\end{array}$} & \multirow{2}{*}{$\begin{array}{l}\text { VEs } \\
(\mathrm{mm}) \\
13,0\end{array}$} & \multirow{2}{*}{$\begin{array}{l}\text { VEd } \\
(\mathbf{m m}) \\
23,0\end{array}$} & \multirow{2}{*}{$\begin{array}{l}\text { MVE } \\
(g) \\
18,0\end{array}$} & \multirow{2}{*}{$\begin{array}{l}\text { IMVE } \\
(\mathrm{g} / \mathrm{m} 2) \\
75,4\end{array}$} & \multirow{2}{*}{$\begin{array}{l}\text { VSVD } \\
(\mathrm{mm}) \\
13,0\end{array}$} & \multirow{2}{*}{$\begin{array}{l}\text { DAo } \\
(\mathrm{mm}) \\
12,0\end{array}$} \\
\hline 0,2 & P50 & & & & & & \\
\hline \multirow{3}{*}{0,3} & P3 & 11,6 & 19,5 & 11,4 & 40,7 & 9,7 & 10,6 \\
\hline & P50 & 14,0 & 23,5 & 20,4 & 62,7 & 13,0 & 13,0 \\
\hline & P97 & 17,4 & 29,0 & 32,9 & 102,8 & 16,0 & 15,0 \\
\hline \multirow{3}{*}{0,4} & P3 & 13,8 & 23,8 & 20,5 & 50,9 & 12,8 & 12,8 \\
\hline & P50 & 16,0 & 28,0 & 29,3 & 69,8 & 16,0 & 15,0 \\
\hline & P97 & 19,4 & 31,9 & 43,5 & 105,2 & 19,4 & 17,0 \\
\hline \multirow{3}{*}{0,5} & P3 & 15,0 & 26,0 & 24,4 & 51,3 & 13,9 & 13,0 \\
\hline & P50 & 18,0 & 30,0 & 35,6 & 68,4 & 18,0 & 15,0 \\
\hline & P97 & 20,2 & 33,1 & 46,9 & 99,7 & 22,1 & 17,1 \\
\hline \multirow{3}{*}{0,6} & P3 & 15,9 & 28,7 & 31,2 & 56,2 & 14,9 & 14,0 \\
\hline & P50 & 19,5 & 32,0 & 43,2 & 74,0 & 18,0 & 16,5 \\
\hline & P97 & 23,9 & 37,0 & 66,5 & 107,0 & 21,3 & 19,1 \\
\hline \multirow{3}{*}{0,7} & P3 & 18,0 & 29,2 & 32,6 & 44,4 & 15,1 & 15,1 \\
\hline & P50 & 21,0 & 35,0 & 50,2 & 71,5 & 18,0 & 17,5 \\
\hline & P97 & 24,0 & 38,0 & 65,8 & 95,9 & 23,7 & 20,8 \\
\hline \multirow{3}{*}{0,8} & P3 & 18,0 & 29,5 & 41,2 & 49,6 & 16,0 & 12,0 \\
\hline & P50 & 21,5 & 37,0 & 60,1 & 77,7 & 20,0 & 19,0 \\
\hline & P97 & 26,0 & 40,5 & 80,3 & 98,6 & 25,5 & 21,3 \\
\hline \multirow{3}{*}{0,9} & P3 & 19,8 & 32,9 & 52,4 & 57,8 & 14,9 & 17,9 \\
\hline & P50 & 22,5 & 38,0 & 69,0 & 74,1 & 20,0 & 20,0 \\
\hline & P97 & 26,1 & 40,0 & 76,5 & 89,1 & 25,1 & 24,0 \\
\hline \multirow{3}{*}{1,0} & P3 & 17,3 & 34,2 & 52,3 & 52,4 & 17,5 & 18,5 \\
\hline & P50 & 24,0 & 40,0 & 77,3 & 76,0 & 21,0 & 21,0 \\
\hline & P97 & 26,9 & 42,9 & 112,0 & 109,9 & 24,0 & 25,0 \\
\hline \multirow{3}{*}{1,1} & P3 & 18,5 & 34,0 & 59,5 & 53,9 & 19,5 & 19,5 \\
\hline & P50 & 24,0 & 41,0 & 78,8 & 72,3 & 23,0 & 21,0 \\
\hline & P97 & 31,0 & 43,5 & 99,7 & 88,5 & 27,5 & 23,5 \\
\hline \multirow{3}{*}{1,2} & P3 & 22,0 & 39,3 & 85,4 & 69,1 & 17,1 & 19,5 \\
\hline & P50 & 24,5 & 42,0 & 100,7 & 82,1 & 22,0 & 22,0 \\
\hline & P97 & 28,5 & 47,0 & 126,5 & 104,2 & 28,7 & 24,0 \\
\hline \multirow{3}{*}{1,3} & P3 & 24,1 & 39,8 & 88,1 & 37,9 & 20,3 & 19,5 \\
\hline & P50 & 24,5 & 42,0 & 110,8 & 70,7 & 24,5 & 23,0 \\
\hline & P97 & 28,0 & 47,4 & 126,2 & 82,3 & 28,8 & 25,0 \\
\hline \multirow{3}{*}{1,4} & P3 & 24,4 & 43,1 & 101,2 & 74,6 & 20,3 & 21,0 \\
\hline & P50 & 28,5 & 45,5 & 120,6 & 86,8 & 23,0 & 23,5 \\
\hline & P97 & 29,0 & 47,9 & 128,0 & 92,9 & 29,4 & 26,0 \\
\hline \multicolumn{8}{|c|}{$\begin{array}{l}\text { SC- superfície corporal; VEs- diâmetro sistólico do ventrículo esquerdo; } \\
\text { VEd- diâmetro diastólico do ventrículo esquerdo; MVE- massa do } \\
\text { ventrículo esquerdo; IMVE- índice de massa do ventrículo esquerdo; } \\
\text { VSVD- diâmetro da via de saída do ventrículo direito; DAo- diâmetro } \\
\text { da aorta. }\end{array}$} \\
\hline \multicolumn{8}{|c|}{$\begin{array}{c}\text { Tabela } 5 \text { - Percentis } 3 \%, 50 \% \text { e } 97 \% \text { dos valores das medidas } \\
\text { ecocardiográficas que apresentaram diferenças estatisticamente } \\
\text { significativas entre os sexos, em relação à superfície corporal das } \\
\text { crianças do sexo feminino }\end{array}$} \\
\hline
\end{tabular}




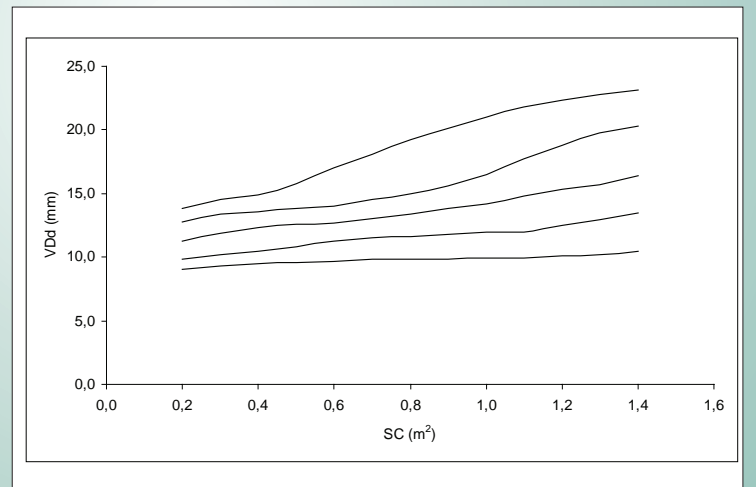

Fig. 3 - Curvas de percentis do diâmetro diastólico do ventrículo direito (VDd) em relação à superfície corporal (SC).

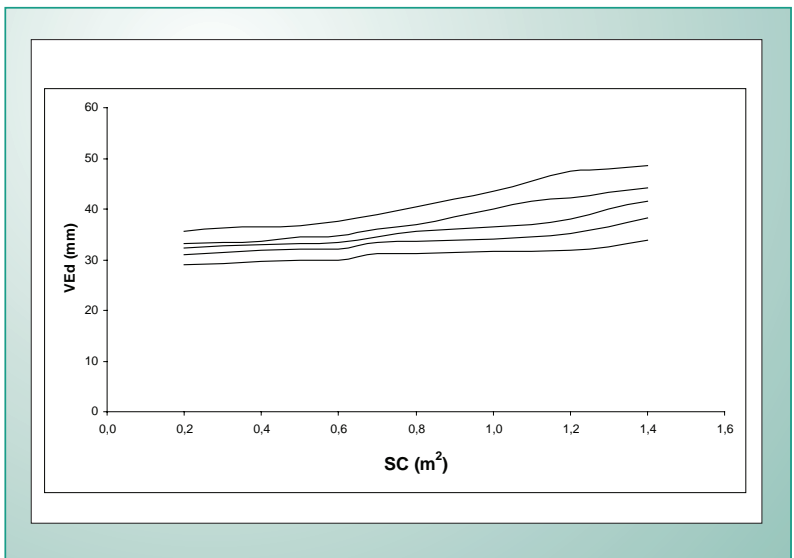

Fig. 5 - Curvas de percentis do diâmetro diastólico do ventrículo esquerdo (VEd) em relação à superfície corporal (SC).

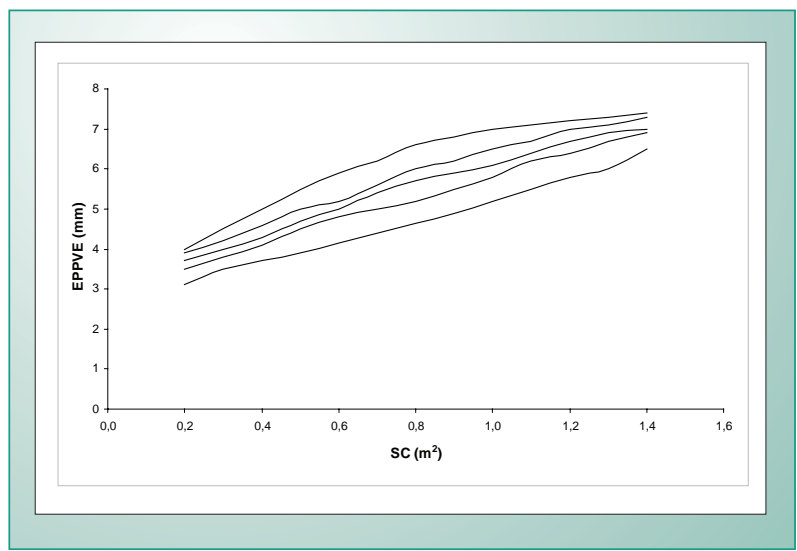

Fig. 7 - Curvas de percentis da espessura diastólica da parede posterior do ventrículo esquerdo (EPPVE) em relação à superfície corporal (SC).

ecocardiográficas que obtivemos também são superponíveis. Kampmann e cols. ${ }^{13}$ obtiveram coeficientes de correlação com valores mais elevados que os verificados neste estudo. No entanto, aqueles autores utilizaram apenas os valores correspondentes ao percentil 50\% da variável ecocardiográfica para o cálculo dos coeficientes de correlação, introduzindo

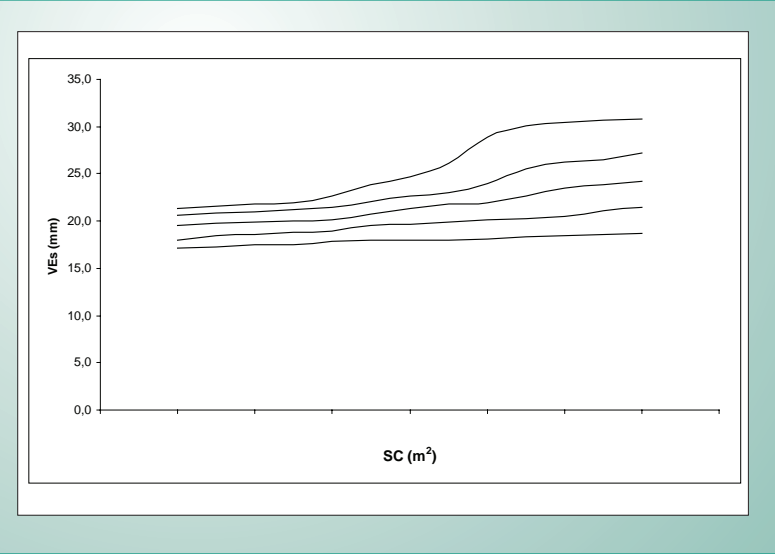

Fig. 4 - Curvas de percentis do diâmetro sistólico do ventrículo esquerdo (VEs) em relação à superfície corporal (SC).

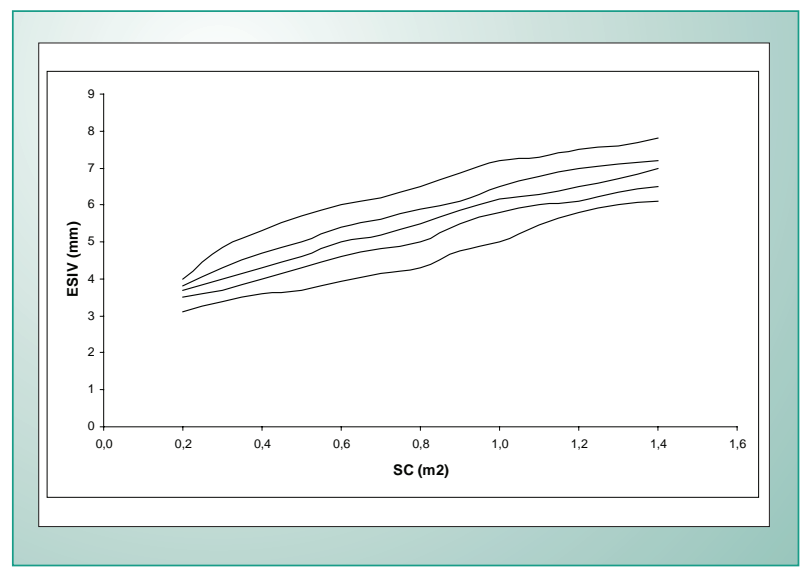

Fig. 6 - Curvas de percentis da espessura diastólica do septo interventricular (ESIV) em relação à superfície corporal (SC).

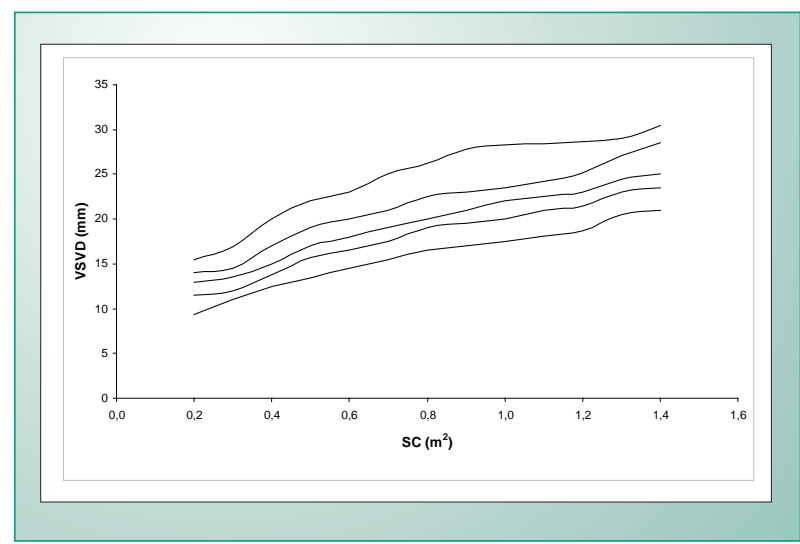

FIG. 8 - CURVAS DE PERCENTIS DO DIÂMETRO DA VIA DE SAÍDA DO VENTRÍCULO DIREITO (VSVD) EM RELAÇÃO À SUPERFÍCIE CORPORAL (SC).

potencial viés, posto que a dispersão dos dados foi excluída do cálculo do coeficiente de correlação.

Nós calculamos as correlações levando em conta todos os valores individuais de cada variável ecocardiográfica e também obtivemos boa correlação para várias medidas ecocardiográficas, principalmente para MVE, VEd, DAo, 


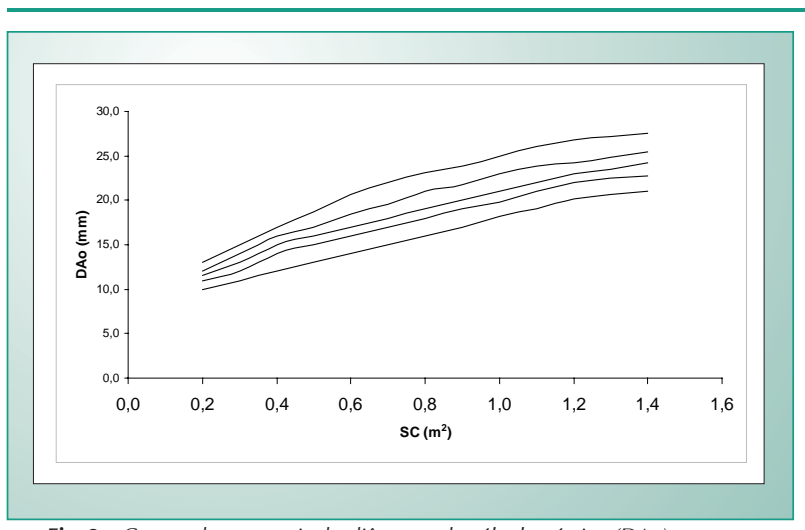

Fig. 9 - Curvas de percentis do diâmetro da válvula aórtica (DAo) em relação à superfície corporal (SC).

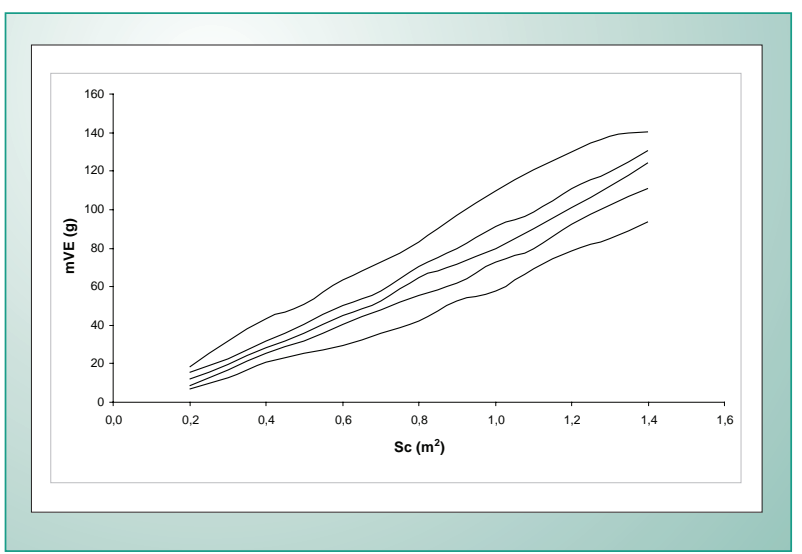

Fig. 11 - Curvas de percentis da massa muscular do ventrículo esquerdo (mVE) em relação à superfície corporal (SC).

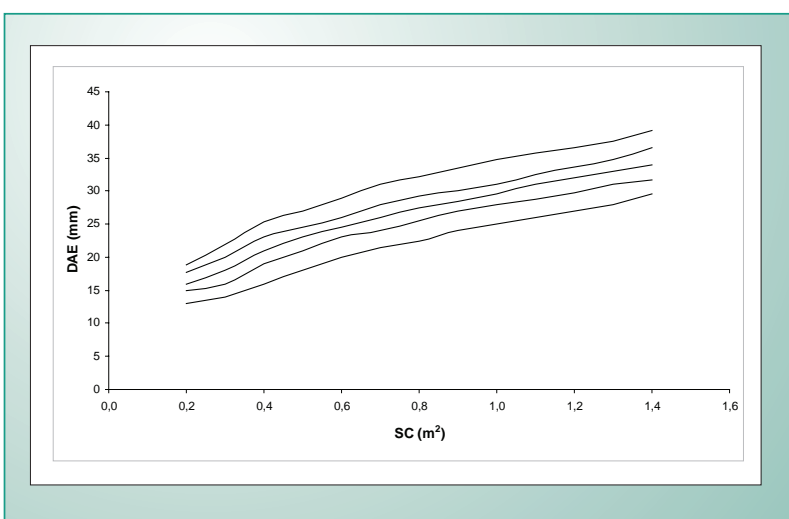

Fig. 10 - Curvas de percentis do diâmetro do átrio esquerdo (DAE) em relação à superfície corporal (SC).

EPPVE, VEs, DAE e ESIV. Não observamos diferenças significantes quando os valores dos coeficientes de correlação de Pearson, obtidos com a utilização de todas as crianças do estudo, foram comparados com aqueles verificados quando se analisam as crianças separadamente de acordo com o sexo. Esse achado evidencia que, apesar do fato dos valores de algumas medidas ecocardiográficas apresentarem diferenças significantes, quando as crianças são separadas de acordo com o sexo, essas diferenças não modificaram significantemente os valores dos coeficientes de correlação de Pearson.

Os percentis 3\% e 97\% foram escolhidos como limite inferior e superior, respectivamente, para a construção das curvas de percentis, visto que correspondem, aproximadamente, à média mais ou menos dois desvios-padrão, de acordo com a curva de distribuição de Gauss ${ }^{40}$. Além disso, foram construídas curvas de percentis de $25 \%, 50 \%$ e $75 \%$, com o objetivo de que, no futuro, essas curvas possam ser utilizadas para acompanhar o remodelamento cardíaco durante tratamento clínico de crianças com cardiopatia congênita ou adquirida, bem como após correção cirúrgica delas, ou acometimento cardíaco decorrente de outras doenças, tais como desnutrição, obesidade e hipertensão arterial sistêmica.

Considerando que do ponto de vista estatístico foram observadas diferenças significantes entre os sexos para as variáveis ecocardiográficas VEs, VEd, VSVD, DAo, MVE e IMVE, os valores dos percentis 3\%, 50\% e 97\% dessas variáveis relacionadas com a SC foram apresentados em tabelas separadas (tabs. 4 e 5).

Implicações clínicas - Este é um estudo de efetividade, pois foi realizado em situações que ocorrem no dia-a-dia, em crianças portadoras de sopro cardíaco, que foram avaliadas para excluir cardiopatia.

Os resultados verificados adicionam e contribuem para melhor conhecimento dos limites inferior e superior das dimensões cardíacas e índices de desempenho cardíaco obtidos por meio da ecocardiografia em crianças eutróficas na faixa etária de 1 a 144 meses.

Considerando-se que os valores das medidas e dos índices de desempenho cardíaco foram estabelecidos a partir de crianças eutróficas sem cardiopatia, pode-se admitir que esses possam ser utilizados como parâmetros de referência durante acompanhamento de crianças com cardiopatia, bem como daquelas submetidas a tratamento cirúrgico. Adicionalmente, os dados são úteis para avaliações das dimensões cardíacas e do grau de acometimento do coração em crianças portadoras de DEP, obesidade e hipertensão arterial sistêmica, condições atualmente comuns em nosso meio.

As curvas de percentis possibilitam quantificar o grau de desvio da normalidade, permitindo avaliar, no decorrer do tempo, as modificações das características do remodelamento cardíaco em crianças portadoras de cardiopatia primária, bem como aquelas associadas a doenças sistêmicas. Nesse sentido, as curvas de percentis possibilitam quantificar mais precisamente o grau de desvio da normalidade e identificar fatores de risco e prognóstico cardiovascular relacionados a diversas doenças que acometem crianças na faixa etária estudada.

Este trabalho também permite validar para o nosso meio os estudos de Rogé e cols. ${ }^{1}$ e Henry e cols. ${ }^{16}$, os mais utilizados como referência para os valores das medidas ecocardiográficas em nosso país. Adicionalmente, os resultados obtidos possibilitaram a construção de curvas de percentis de valores das medidas cardíacas relacionadas à SC, o que amplia as informações da literatura em relação do estudo de Kampmann e cols. ${ }^{13}$

Em resumo, os resultados observados evidenciaram diferenças estatisticamente significantes entre os sexos das medidas cardíacas: VEs, VEd, MVE, IMVE, VSVD e DAo, indicando que, em crianças, o sexo deve ser considerado 
quando se analisam medidas ecocardiográficas. Os valores encontrados são superponíveis aos relatados em outros grupos étnicos de crianças eutróficas, na mesma faixa etária. As curvas de percentis construídas neste estudo ampliam os dados disponíveis na literatura e possibilitam acompanhamento da remodelação cardíaca de crianças com cardiopatias e/ou doenças sistêmicas que envolvem o coração.

\section{Referências}

1. Rogé $\mathrm{CL}$, Silverman NH, Hart PA, Ray RM. Cardiac structure growth pattern determined by echocardiography. Circulation. 1978;57:285-90.

2. Phoon CKL, Divekar A, Rutkowski M. Pediatric echocardiography: applications and limitations. Curr Probl Pediat. 1999;29:162-85.

3. Henry WL, Ware J, Gardin JM, Hepner SI, McKay J, Weiner M. Echocardiographic measurements in normal subjects. Growth-related changes that occur between infancy and early adulthood. Circulation. 1978;57:278-85.

4. Feigenbaum H. Echocardiography. 3rd ed. Philadelphia: Lea \& Febiger, 1981.

5. Schneider C, Cabizuca SV, Benchimol CB, Matsunaga LA, Ginefra P, Albanez Filho FA, et al. Padrões ecocardiográficos normais em crianças da cidade do Rio de Janeiro. I. Relação entre medidas ecocardiográficas e padrões antropométricos. Arq Bras Cardiol. 1986;47:139-92.

6. Huwez FU, Houston AB, Watson J, McLaughlin S; Macfarlane PW. Age and body surface area related to normal upper and lower limits of $\mathrm{M}$ mode echocardiographic measurements and left ventricular volume and mass from infancy to early adulthood. Br Heart J. 1994;72:276-80.

7. Devereux RB, Liebson PR; Horan MJ. Recommendations concerning use of echocardiography in hypertension and general population research. Hypertension. 1987; 9 (Suppl. II): 97-104

8. Savage DD, Garrison RJ, Kannel WB, Andersosn SJ. Considerations in the use of echocardiography in epidemiology: The Framingham Study. Hypertension. 1987;9(Suppl. II): 40-44.

9. Ascione L, De Leva F, Cuomo S, Caso MSP, Cioppa L, Mininni N. Reference values for the echocardiographic calculation of left ventricular mass in normal children between the ages of 0 and 6 years. G Ital Cardiol. 1992;22:829-34.

10. Dai S, Ayres NA, Harrist, RB, Bricker T, Labarthe DR. Validity of echocardiographic measurement in an epidemiological study - Project HeartBeat. Hypertension. 1999;34:236-41.

11. Kampmann C. Echokardigrafishce Normwerte im Kinderslater. In: Schmali|z KJG, ed. Kardiale Ultraschalldiagmostik, Handbuch und Atlas. Berlin: Blackwell Wissenschaft, 1994. p.389-91.

12. Schvartzman PR, Fucks FD, Mello AG, Coli M, Schvartzman M, Moreira LB. Valores normais de medidas ecocardiográficas. Um estudo populacional. Arq Bras Cardiol. 2000;75:107-14.

13. Kampmann C, Wiethoff CM, Wenzel A, Stolz G, Betancor M, Wippermann $\mathrm{CF}$, et al. Normal values of $\mathrm{M}$ mode echocardiographic measurements of more than 2000 healthy infants and children in central Europe. Heart. 2000;83:667-72.

14. Epstein ML, Goldberg ST, Allen HD, Konecke L, Wood J. Great vessel, cardiac chamber and wall growth patterns in normal children. Circulation. 1975;51:1124-9.

15. Gutgesell HP, Paquet M, Duff DR, McNamara DG. Evaluation of left ventricular size and function by echocardiography. Results in normal children. Circulation. 1977;56:457-62.

16. Henry WL, Gardin JM, Ware JH. Echocardiographic measurements in normal subjects from infancy to old age. Circulation. 1980;62:1054-60.

17. Schneider C, Cabizuca SV, Benchimol CB, Matsunaga LA, Ginefra P, Albanesi Filho FM, et al. Padrões ecocardiográficos normais em crianças da cidade do Rio de Janeiro. II. Medidas dos diâmetros, dimensões, espessuras de estruturas cardíacas e movimentos valvulares. Arq Bras Cardiol. 1986;47:253-8
Financiamento: $\mathrm{CNPq}$ - Bolsa de Iniciação Científica (Bolsa PIBIC).

\section{Potencial Conflito de Interesses}

Declaro não haver conflitos de interesses pertinentes.

18. Schneider C, Cabizuca SV, Benchimol CB, Matsunaga LA, Ginefra P, Albanes Filho FM, et al. Padrões ecocardiográficos normais em crianças da cidade do Rio de Janeiro. III. Avaliação das dimensões e funções do ventrículo esquerdo. Arq Bras Cardiol. 1986; 47:335-40.

19. DerSimoniam R, Levine RJ. Resolving discrepancies between a meta-analysis and a subsequent large controlled trial. JAMA. 1999;282:664-70.

20. Morcef FAP. Ecocardiografia uni-bidimensional, transesofágica e Doppler 2a ed. Rio de Janeiro: Livraria e Editora Revinter; 2001.

21. Martins TC, Soares AM. Ecocardiograma normal em crianças. In: Ramires JAF ed. Cardiologia em Pediatria: temas fundamentais. São Paulo: Roca; 2000. p. 53-81.

22. Marcondes E, Berquó ES, Yunes J, Luongo J, Martins JS. Estudo antropométrico de crianças brasileiras de zero a doze anos de idade. Anais Nestlé. 1971; 84: $10-5$

23. DuBois D, DuBois EF. A formula to estimate the approximate surface area if height and weight be known. Arch Intern Med. 1916;17:863-71.

24. Garrow JS, Webster J. Quetelet's index $(\mathrm{W} / \mathrm{H} 2)$ as a measure of fatness. Int J Obes. 1985;9:147-53.

25. World Health Organization. Physical status: the use and interpretation of antropometry. Genebra; 1995. Technical Report Series 854.

26. Must A, Dallal GE, Dietz WH. Reference data for obesity: 85th and 95th percentiles of body mass index (wt/ht2) - a correction. Am J Clin Nutr. $1991 ; 54: 773$.

27. Singh GR, Malathi KE, Kasliwal RR, Ommar A, Padmavati S, Ramji S. An evaluation of cardiac function in malnourished children by non-invasive methods. Indian Pediatr. 1989; 26:875-81.

28. Kothari SS, Patel TM, Shetalwad AN, Patel TK. Left ventricular mass and function in children with severe protein energy malnutrition. Int J Cardiol. 1992:35:19-25

29. Phornphatkul C, Pongprot Y, Suskind R, George V, Fuchs G. Cardiac function in malnourished children. Clin Pediatr. 1994;33:147-54.

30. Gelb BD, Abdenur J. Metabolic heart disease. In: Garson A, Bricker TJ, Fishe DJ, Neish JR (eds.). The science and practice of pediatric cardiology.2nd ed. Baltimore: Williams \& Wilkins; 1998. p. 1913.

31. Öcal B, Ünal S, Zorlu P, Tezic HT, Oguz D. Echocardiographic evaluation of cardiac functions and left ventricular mass in children with malnutrition. J Paediatr Child Health. 2001; 37:14-7.

32. Okoshi MP, Okoshi K, Pai VD, Pai-Silva MD, Matsubara LS, Cicogna AC Mechanical, biochemical, and morphological changes in the heart from chronic food-restricted rats. Can J Physiol Pharm. 2001;79:754-60.

33. Fioretto JR, Queiroz SS, Padovani CR, Matsubara LS, Okoshi K, Matsubara BB. Ventricular remodeling and diastolic myocardial dysfunction in rats submitted to protein-calorie malnutrition. Am J Physiol Heart Circ Physiol. $2002 \cdot 282 \cdot \mathrm{H} 1327-33$

34. de Simone G, Daniels SR, Devereux RB, Meyer RA, Roman MJ, De Vittis $\mathrm{O}$, et al. Left ventricular mass and body size in normotensive children and adults: Assessment of allometric relations and impact of overweight. J Am Coll Cardiol. 1992;20:1251-60.

35. Popp RL. Echocardiographic assessment of cardiac disease. Circulation. 1976;54:538-41.

36. Sahn DJ, DeMaria A, Kisslo J, Weyman A. The Committee on M Mode standardization of the American Society of Echocardiography. 
Bonatto e cols.

CURVAS DE PERCENTIS DE VALORES NORMAIS DE MEDIDAS ECOCARDIOGRÁFICAS EM

CRIANÇAS EUTRÓFICAS PROCEDENTES DA REGIÃO CENTRO-SUL DO ESTADO DE SÃO PAULO

Artigo Original

Recommendations regarding quantitation in M-mode Echocardiography: results of a survey of echocardiographic measurements. Circulation. 1978;58:1072-83.

37. Coté CJ. Sedação no paciente pediátrico - uma revisão. In: Wetzel RC, ed. Clínicas Pediátricas da América do Norte. Rio de Janeiro: Interlivros; 1994. p. 35-66.

38. Devereux RB, Reichek N. Echocardiographic determination of left ventricular mass in man: anatomic validation of the method. Circulation. 1977;55:613-8.

39. Pombo JF, Troy BL, Russel RO Jr. Left ventricular volumes and ejection fraction by echocardiography. Circulation. 1971;43:26-33.

40. Zar JH. Biostatistical analysis. 4th ed. New Jersey: Prentice-Hall, 1999.

41. Lester LA, Sodt PC, Hutcheon N. M-mode echocardiography in normal children and adolescents: some new perspectives. Pediatr Cardiol. 1987;8:27-33.

42. Nagasawa H, Arakaki Y, Yamada O, Nakajima T, Kamiya T. Longitudinal observations of left ventricular end-diastolic dimension in children using echocardiography. Pediatr Cardiol. 1996; 17:169-74.

43. Nagasawa $\mathrm{H}$, Arakaki Y. Identification of gender differences in the thickness of the left ventricular wall by echocardiography in children. Cardiol Young. 2002; 12:37-43. 